\title{
Protein complex points to new route to Rett syndrome
}

\section{BY ANGIE VOYLES ASKHAM}

18 FEBRUARY 2022

Researchers have identified a new protein complex that shapes the function of the MECP2 protein, encoded by the gene mutated in most cases of Rett syndrome. The complex reveals a novel pathway underpinning the syndrome's traits and a potential target for treatment, the researchers say.

Children with Rett syndrome typically experience breathing and motor problems, repetitive behaviors and a regression in speech and social skills. About 95 percent carry genetic mutations that impair MECP2 protein, which regulates gene expression by binding to regions of DNA marked by chemical tags called methyl groups.

MECP2 protein also binds to a complex containing the protein NCOR1, which regulates chromatin - the conglomerate of DNA and proteins that makes up chromosomes. Mutations that disrupt MECP2 protein's ability to bind DNA usually lead to severe Rett syndrome, but those that abolish its ability to link up with NCOR1 cause relatively milder Rett traits, says lead investigator Huda Zoghbi, professor of molecular and human genetics at Baylor College of Medicine in Houston, Texas. "That told us that maybe there are other interactors" that can contribute to the condition, she says.

In the new work, Zoghbi and her colleagues identified another interactor: TCF20, a protein that, in forming a complex with MECP2, facilitates DNA binding and regulates gene expression. Mutations in TCF20 can disrupt its ability to bind to MECP2 and lead to Rett-like traits in mice, the team found.

The findings show that "the TCF20 complex is clearly important for MECP2-dependent gene regulation, and needs to be taken into consideration when studying [Rett syndrome]," says Hume Stroud, assistant professor of neuroscience at the University of Texas Southwestern Medical Center in Dallas, who was not involved in the work. 


\section{Spectrum | Autism Research News}

https://www.spectrumnews.org

Zoghbi and her colleagues went hunting for proteins that interact with MECP2 using a method called BiolD, which flagged any molecule that came into close proximity with the Rett protein.

Because BiolD captures "potentially weaker, and more transient, interactions," it can identify candidates that "that could be missed using more typical biochemical approaches," says Lisa Boxer, Stadtman investigator in the Laboratory of Genome Integrity at the National Institutes of Health, who was not involved in the study.

In addition to gathering a list of all proteins that seem to interact with MECP2 protein, the researchers used the method to spot proteins that link up with two mutated forms of MECP2 protein - one that cannot enter a cell's nucleus and one that cannot bind DNA. Proteins that interact with the standard form, but not the mutated ones, are likely to bind to chromatin and contribute to MECP2 protein's function, says study investigator Jian Zhou, a postdoctoral researcher in Zoghbi's lab. One such protein is TCF20, the team found. The findings were published in the Proceedings of the National Academy of Sciences in January.

TCF20, along with another protein, PHF14, seem to form a complex with MECP2 protein, Zhou says. All three are present together in neurons and expressed at similar times, he and his colleagues found. And both MECP2 and TCF20 proteins regulate expression of the gene that codes for brain-derived neurotrophic factor (BDNF), a protein that encourages the growth of new synapses, the researchers found through gene expression analysis.

Mice engineered to have decreased expression of TCF20 have few anxiety-like behaviors, poor performance on learning and memory tasks, and altered social behaviors, the team found - similar to what is seen in a mouse model of Rett syndrome. Breeding them with mice that have excess MECP2 expression produced offspring with fewer atypical behaviors, suggesting that "at least part of the behavioral deficit caused by MECP2 duplications, or too much MECP2, is through TCF20," Zhou says.

Past research has suggested that mutations in the TCF20 protein complex lead to Rett-like traits in people, too. One study identified a woman with Rett syndrome who has a mutation in TCF20 rather than MECP2 - pointing to the shared mechanism.

But the picture is complicated by the other protein in the complex, PHF14, Zoghbi says. She and her colleagues identified a person with intellectual disability who carries mutations in PHF14 that disrupt the interaction between MECP2 and the rest of the complex. But other individuals with PHF14 mutations have no Rett traits. And mice engineered to have decreased expression of PHF14 do not have altered behaviors, the team found.

"At this point, it's safe to say, if mutations in PHF14 cause a human disorder, they're not completely penetrant," Zoghbi says. 


\section{Spectrum | Autism Research News}

https://www.spectrumnews.org

Still, PHF14 and TCF20 warrant additional follow up, Boxer says. "It would be great to find additional patients with mutations in these genes to verify that [they're] really causative of the disease, and then potentially make mouse models with this specific mutation to try it out."

Zogbhi says she and her colleagues plan to look into why mutations in PHF14 have these different effects. They also plan to investigate whether there are points of convergence for other neurodevelopmental conditions within this pathway.

"There may be a class of disorders [that] share some biology, so that maybe eventually if you can have a treatment that works for one of them, it may be helpful to the whole class," Zoghbi says.

BDNF, which is known to be relevant for Rett syndrome, might represent one such shared pathway, says Qiang Chang, professor of medical genetics and neurology at University of Wisconsin-Madison, who was not involved in the work. Although targeting BDNF has not panned out as a treatment for Rett syndrome, he says, these findings offer an upstream target in the same pathway that may prove useful.

"Therapeutically, knowing [about] this new pathway, this new complex, is really informative," Chang says.

Cite this article: https://doi.org/10.53053/PLVD5194 